\title{
Global Facilitation of Attended Features Is Obligatory and Restricts Divided Attention
}

\author{
Søren K. Andersen, ${ }^{1,2,3}$ Steven A. Hillyard, ${ }^{2}$ and Matthias M. Müller ${ }^{3}$ \\ ${ }^{1}$ School of Psychology, University of Aberdeen, Aberdeen AB24 3FX, United Kingdom, ${ }^{2}$ Department of Neurosciences, University of California, San Diego, \\ La Jolla, California 92093, and '3nstitute of Psychology, University of Leipzig, 04103 Leipzig, Germany
}

In many common situations such as driving an automobile it is advantageous to attend concurrently to events at different locations (e.g., the car in front, the pedestrian to the side). While spatial attention can be divided effectively between separate locations, studies investigating attention to nonspatial features have often reported a "global effect", whereby items having the attended feature may be preferentially processed throughout the entire visual field. These findings suggest that spatial and feature-based attention may at times act in direct opposition: spatially divided foci of attention cannot be truly independent if feature attention is spatially global and thereby affects all foci equally.

In two experiments, human observers attended concurrently to one of two overlapping fields of dots of different colors presented in both the left and right visual fields. When the same color or two different colors were attended on the two sides, deviant targets were detected accurately, and visual-cortical potentials elicited by attended dots were enhanced. However, when the attended color on one side matched the ignored color on the opposite side, attentional modulation of cortical potentials was abolished. This loss of feature selectivity could be attributed to enhanced processing of unattended items that shared the color of the attended items in the opposite field. Thus, while it is possible to attend to two different colors at the same time, this ability is fundamentally constrained by spatially global feature enhancement in early visual-cortical areas, which is obligatory and persists even when it explicitly conflicts with task demands.

\section{Introduction}

Studies of visual-spatial attention have revealed substantial flexibility in dividing attention between stimuli at different spatial locations (Pylyshyn and Storm, 1988; Awh and Pashler, 2000; Müller et al., 2003). In contrast, the processing of an attended stimulus feature such as a color, orientation, or direction of motion may be facilitated across the entire visual field, even when only a portion of the field needs be attended (Treue and Martínez Trujillo, 1999; Saenz et al., 2002, 2003; Martinez-Trujillo and Treue, 2004; Lu and Itti, 2005; Melcher et al., 2005). Such a spatially "global" mechanism is highly useful when searching for objects defined by single features or feature conjunctions (Maljkovic and Nakayama, 1994; Wolfe, 1994; Müller et al., 1995; Kristjánsson, 2006; Andersen et al., 2008; Zhou and Desimone, 2011). However, this global facilitation may actually impede an observer's ability to attend to a particular feature at one location in the visual field while ignoring that feature in favor of another at a different location. This impediment would be particularly se-

\footnotetext{
Received May 7, 2013; revised Aug. 14, 2013; accepted Aug. 26, 2013.

Author contributions: S.K.A., S.A.H., and M.M.M. designed research; S.K.A. performed research; S.K.A. analyzed data; S.K.A., S.A.H., and M.M.M. wrote the paper.

This work was supported by the Deutsche Forschungsgemeinschaft (AN 841/1-1, MU 972/20-1), the U.S. National Institute of Mental Health (1P50MH86385), the National Science Foundation (BCS-1029084), and the Office of Naval Research (N00014-07-1-0937). We thank Renate Zahn, Sophie Trauer, Christian Keitel, and Jennifer Padwal for help with data collection and Matt Marlow for technical support.

The authors declare no competing financial interests.

Correspondence should be addressed to Søren K. Andersen, School of Psychology, University of Aberdeen, William Guild Building, Kings College, Aberdeen AB24 3FX, UK. E-mail: skandersen@abdn.ac.uk.

DOI:10.1523/JNEUROSCI.1913-13.2013

Copyright $\odot 2013$ the authors $\quad 0270-6474 / 13 / 3318200-08 \$ 15.00 / 0$
}

vere if global facilitation of attended features were (a) obligatory, in that it occurred regardless of whether it was beneficial or detrimental to the task at hand, and (b) uniform, in that the facilitation would be of equal magnitude across the visual field rather than a decrementing spread with a reduced effect at more distant locations.

Despite intensive investigations of feature-based visual attention in recent years, evidence for whether its spatially global effect is obligatory and/or uniform remains surprisingly sparse. In a typical experimental design, participants attended to a stimulus having the relevant feature on one side of a central fixation point while measuring the physiological brain response to a taskirrelevant stimulus placed on the opposite side. Enhanced neural processing of the stimulus on the unattended side was generally observed when it shared the relevant feature with the contralateral attended stimulus as opposed to when it did not (Treue and Martínez Trujillo, 1999; Saenz et al., 2002; Martinez-Trujillo and Treue, 2004; Serences and Boynton, 2007; Stoppel et al., 2007, 2012; Hayden and Gallant, 2009; Zhang and Luck, 2009; Andersen et al., 2011a; Bondarenko et al., 2012). This type of paradigm, however, does not address the key question of whether or not the observed global effect is obligatory. Moreover, these previous studies typically obtained only behavioral measures of feature selectivity on the attended side, and thus were not informative about whether facilitation of the relevant feature was uniform across the entire visual field. A behavioral study where attention was divided between left and right visual fields found interference between competing features that was suggestive of an obligatory global enhancement (Saenz et al., 2003), but it was not clear 


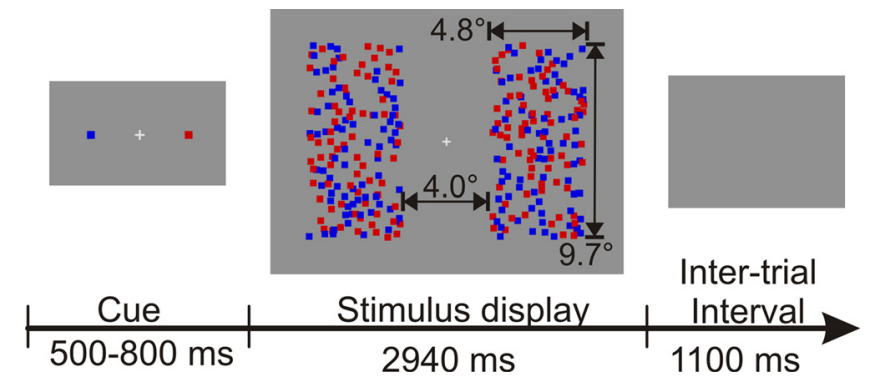

Figure 1. Schematic illustration of the time course of an individual trial. Stimulus size in visual angles is indicated by numbers and arrows that were not present in the experiment.

whether or not such enhancement was uniform across the visual field.

The present study combines electrophysiological recordings of frequency-tagged potentials and behavioral measures in a divided attention task to test the hypothesis that global enhancement of feature-selective processing is an obligatory and spatially uniform property of human attention that persists even when it conflicts with task demands.

\section{Materials and Methods}

In two experiments, participants viewed two fields, each consisting of randomly moving intermingled dots of two different colors, one on either side of central fixation (Figs. 1, 2A). Three different experimental conditions were contrasted, in which participants attended to arrays of random dots of the same color (Experiment 1, "attend same," left red and right red or left blue and right blue), opposite colors (Experiment 1, "attend opposite," left red and right blue or left blue and right red) or different colors (Experiment 2, "attend different," e.g., attend left orange and right magenta; Fig. $3 A$ ) on the two sides, respectively. The assigned task in all conditions was to detect brief luminance decrements of $20 \%$ of the dots of either of the attended arrays (targets) while ignoring equivalent luminance decrements of the unattended arrays of dots (distractors).

Steady-state visual evoked potentials (SSVEPs) were used as measures of feature-selective processing of both attended and unattended stimuli in early visual-cortical areas. The SSVEP is an oscillatory response of the visual cortex having the same frequency as the driving stimulus and an amplitude that is enhanced by attending to a feature of the driving stimulus (Andersen et al., 2011b). Presenting stimuli concurrently that flicker at different frequencies allows for the simultaneous measurement of the allocation of attention to each of these "frequencytagged" stimuli, even when they are spatially overlapping (Morgan et al., 1996; Di Russo et al., 2002; Andersen et al., 2011b). In the present study, each of the four fields of dots flickered at a different frequency, thereby driving separable SSVEPs.

While global feature facilitation should be highly effective at enhancing the processing of attended-color stimuli in the "attend-same" and "attend-different" conditions, this should not be the case for the "attendopposite" conditions, because paying attention to a particular color on one side would globally facilitate the same unattended feature on the other side, thereby canceling any selectivity. Indeed, if the global feature facilitation were uniform across the entire field, this should produce a complete cancellation of selectivity as reflected in the SSVEP. On the other hand, if global feature facilitation could be overridden by the task requirements or were reduced in magnitude at a distant location, we would expect to find selective enhancement of the SSVEPs to the explicitly attended dot arrays relative to the unattended arrays under all conditions.

\section{Experiment 1}

Subjects. Fourteen subjects (10 female; 13 right-handed; ages 21 to 31; average, 24.0 years) with normal color perception and normal or corrected-to-normal visual acuity participated in the experiment after giving informed consent. One participant reported problems maintain- ing concentration and fixation during the recording and correspondingly showed very poor task performance and was excluded from final analysis. The experiment was conducted in accordance with the Declaration of Helsinki.

Procedure. In Experiment 1, two completely overlapping fields of randomly moving dots, one red and the other blue in color, were presented concurrently in both the left and right visual fields (Fig. 2A). Participants performed a divided attention task, in which they attended concurrently to one of the overlapping fields on the left and one on the right. We compared conditions in which both attended fields of dots had the same color with conditions where the attended colors were opposite (i.e., the attended color on one side was the same as the unattended on the other side and vice versa). Each of the four fields of dots flickered at an individual frequency synchronized to the screen's refresh rate (blue left, 7.50 $\mathrm{Hz}$; red right, $8.57 \mathrm{~Hz}$; red left, $10.00 \mathrm{~Hz}$; blue right, $12.00 \mathrm{~Hz}$ ), and enhanced cortical processing of attended dot fields was assessed by means of SSVEPs elicited by these frequency-tagged stimuli (Fig. 2A).

Each trial started with the presentation of a central fixation cross and a color cue (Fig. 1). The color cue consisted of one dot on the left and one dot on the right side of the fixation cross, which directly indicated the to-be-attended color on each side for that trial. After a random interval of 500 to $800 \mathrm{~ms}$, the color cue was removed and the flickering dot arrays were presented for $2940 \mathrm{~ms}$ followed by an intertrial interval of $1100 \mathrm{~ms}$, during which only the gray background was presented.

Occasional target and distractor stimuli consisted of brief intervals (200 ms) when a random $20 \%$ of the dots of one color on one side became $30 \%$ dimmer. These luminance decrements could occur randomly in either one of the two attended fields of dots (targets) or in one of the two unattended fields (distractors).

Stimulus material. Stimulation was presented on a 19 inch computer monitor set to a resolution of $640 \times 480$ pixels, 32 bits per pixel color mode, and a refresh rate of $120 \mathrm{~Hz}$. Before EEG recordings, isoluminance of dots and gray background $\left(9.3 \mathrm{~cd} / \mathrm{m}^{2}\right)$ was adjusted for each subject by means of heterochromatic flicker photometry (Wagner and Boynton, 1972). At a viewing distance of $80 \mathrm{~cm}$, each of the four arrays of 75 dots formed a rectangle with a size of $4.8 \times 9.7^{\circ}$ of visual angle centered at the horizontal meridian (Fig. 1). The inner edges were located $2.0^{\circ}$ to the left or right of fixation. Single dots had a size of $0.32^{\circ}$ and moved $0.05^{\circ}$ in a random direction at each frame of screen refresh. All dots were drawn in random order to prevent systematic overlapping of dots of different colors, which might otherwise have induced a depth cue. Stimulation was realized using Cogent Graphics (J. Romaya, Laboratory of Neurobiology, Wellcome Department of Imaging Neuroscience, University College London, London, UK)

The experiment consisted of 560 trials distributed over eight blocks of 70 trials each. Between one and three luminance decrement targets and distractors were presented on a randomly distributed 240 of the trials, while the remaining 320 trials contained none. This resulted in a total of 60 targets and 60 distractors for each attentional condition. The earliest onset of targets and distractors was $500 \mathrm{~ms}$ after onset of the moving and flickering dots, and onsets of subsequent targets and distractors were separated by at least $700 \mathrm{~ms}$. Button presses occurring within an interval from 250 to $900 \mathrm{~ms}$ after onset of a target or distractor were counted as hits and false alarms, respectively. Trials having the four different attentional cueing conditions (attend red-red, blue-blue, red-blue, and blue-red) with or without targets or distractors were presented in random order. Before recordings, subjects performed a training session of two or more blocks until stable performance was reached. The responding hand was changed half way through each recording session.

Participants' behavioral performance was quantified in terms of their ability to discriminate between targets and distractors (observer sensitivity $d^{\prime}$ ) and their tendency to respond rather than refraining from doing so (response bias $C$ ) according to the equal-variance Gaussian model of signal detection theory (Wickens, 2002). Sensitivity $d^{\prime}$, response bias $C$, and target reaction times were first calculated separately for each stimulus and then averaged across stimuli of the same type before statistical analysis.

EEG recordings and analysis. Brain electrical activity was recorded noninvasively from $64 \mathrm{Ag} / \mathrm{AgCl}$ electrodes mounted in an elastic cap at a 
sampling rate of $256 \mathrm{~Hz}$ using an ActiveTwo amplifier system (BioSemi). Except for the built-in anti-aliasing filter of the amplifier, no additional filtering was used. Lateral eye movements were monitored with a bipolar outer canthus montage (horizontal electro-occulogram). Vertical eye movements and blinks were monitored with a bipolar montage positioned below and above the right eye. Processing of EEG data was performed using the EEGLab toolbox (Delorme and Makeig, 2004) in combination with custom written procedures in Matlab (MathWorks).

Epochs for SSVEP analysis were extracted on each trial from 400 to $2900 \mathrm{~ms}$ after stimulation onset to exclude the visual evoked potential to onset and to allow the SSVEP sufficient time to build up. Only trials without targets or distractors were analyzed to ensure that attentional selection was based solely on color and location with no influence of luminance decrement intervals. To correct for any linear drifts, data were detrended (removal of mean and linear trends). Trials with eye movements or blinks were rejected, and any remaining artifacts were corrected by means of an automated procedure using a combination of trial exclusion and channel approximation based on statistical parameters of the data (Junghöfer et al., 2000), leading to an average rejection rate of $11.0 \%$ of all trials. Subsequently, SSVEP epochs were rereferenced to the average of all electrodes and averaged separately for each experimental condition.

SSVEP amplitudes for each electrode and flicker frequency were quantified as the absolute value of the complex Fourier coefficient. Based on isocontour voltage maps of SSVEP amplitudes averaged over all four attentional conditions (Fig. 2B), a cluster of 11 occipital and parietal electrodes (PO7, $\mathrm{PO} 3, \mathrm{POz}$, PO4, PO8, O1, Oz, $\mathrm{O}_{2}, \mathrm{I} 1, \mathrm{Iz}$, and I2) showing maximal amplitudes was chosen for statistical analysis.

Epochs for event-related potential (ERP) analysis were extracted from $100 \mathrm{~ms}$ before to $600 \mathrm{~ms}$ after onsets of luminance decrement targets and distractors. These ERP epochs were rereferenced to averaged earlobes (averaged mastoids for Experiment 2), artifact corrected in the same way as SSVEP epochs, and low-pass filtered below $45 \mathrm{~Hz}$. The mean amplitude over $100 \mathrm{~ms}$ before stimulus onset was used as a baseline. The $\mathrm{P} 3$ component peaked over electrodes $\mathrm{Pz}$ and POz. Accordingly, amplitudes and latencies of the late positive $\mathrm{P} 3$ component difference waveforms (attended-unattended) were analyzed at electrodes $\mathrm{Pz}$ and $\mathrm{POz}$ for the attend-same, attend-different, and attend-opposite conditions. Comparisons of $\mathrm{P} 3$ peak latencies between conditions were made using a permutation test. For each of the three possible comparisons (same vs opposite, same vs different, opposite vs different), a distribution of the peak latency difference of the grand mean of the attended-unattended P3 was generated by randomly assigning the data to conditions. This procedure was repeated 100,000 times and $p$ values were determined as the proportion of random samples having an equal or larger absolute latency difference than the observed latency difference between conditions.

\section{Experiment 2}

In Experiment 2, brain activity was recorded from 64 scalp sites with an SA Instrumentation amplifier, with bandpass set at 0.1 to $80 \mathrm{~Hz}$ and a sampling rate of $250 \mathrm{~Hz}$. Thirteen paid volunteers were recorded. Two subjects were excluded from analysis due to poor signal-to-noise ratios of their SSVEPs and/or a large number of artifacts. The average rejection rate was $22.6 \%$ of all trials. All other aspects of the recording and analysis were identical to Experiment 1 . The critical difference from Experiment 1 was that dot arrays of four different colors were presented (Fig. $3 A$ ). Magenta and cyan dots were presented on one side of fixation, and orange and violet dots on the other side. These colors were generated by adding $50 \%$ of blue or green (in red-green-blue color-space) to the pure red and blue colors used in Experiment 1. On half of the trials, the assignment of colors to sides was reversed, while maintaining the assignment of flicker frequencies to the two sides.

Analysis of single trial SSVEP amplitudes. A separate single-trial analysis was performed to determine whether subjects were switching attention between the two cued dot arrays or whether they were attending concurrently to both of the cued arrays. SSVEP amplitudes for each stimulus frequency were extracted on each single trial by means of Fourier transform for each of the electrodes of the main analysis. The phaselocked SSVEP amplitudes were then averaged over electrodes. For each condition and subject, the correlation between the amplitudes elicited by the two cued stimuli was calculated. The resulting 88 correlations $(8$ conditions by 11 subjects) were then $z$ transformed and subjected to a two-tailed $t$ test against zero. The same analysis was also performed for a 
A

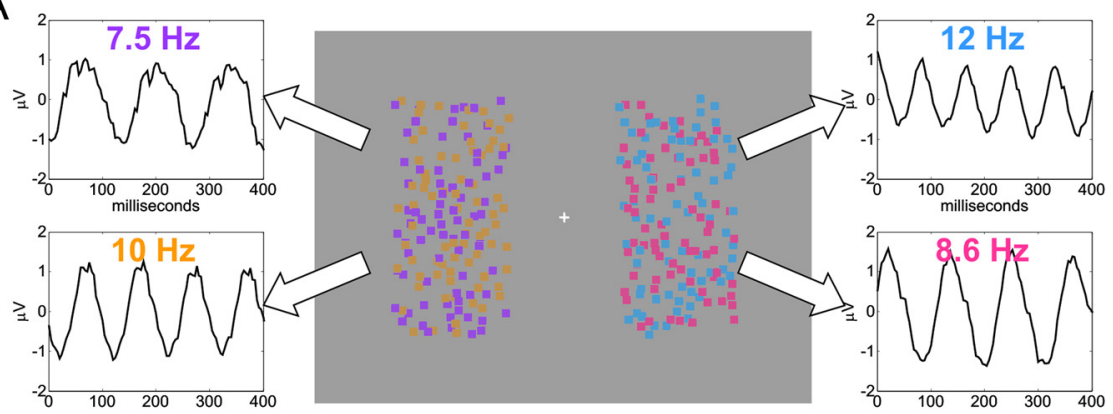

B
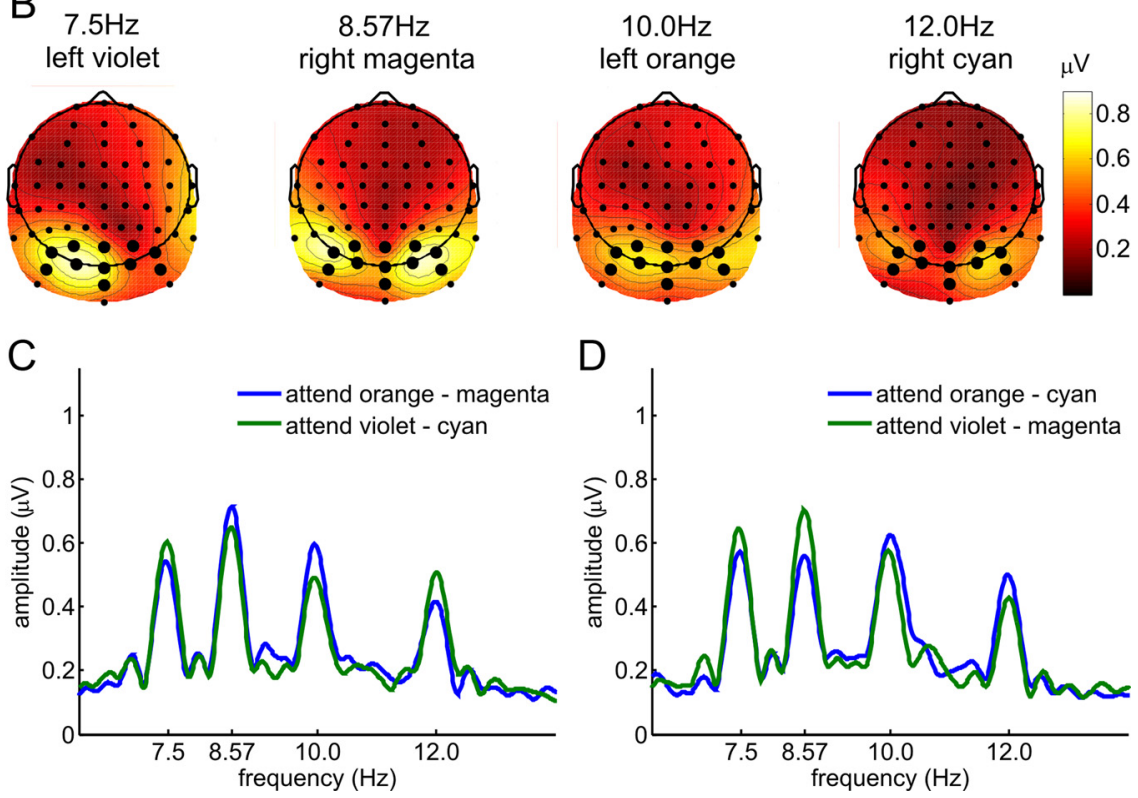

Figure 3. Experiment 2. A, Stimulus display and schematic illustration of assignment of flicker frequencies to stimuli, with SSVEP waveforms obtained from a single subject by moving window averages. All dots were in constant random incoherent motion, and each of the four fields of dots flickered at a specific frequency: violet left, $7.5 \mathrm{~Hz}$; orange left, $10 \mathrm{~Hz}$; cyan right, $12 \mathrm{~Hz}$; magenta right, $8.6 \mathrm{~Hz}$. B , Spline-interpolated isocontour voltage maps of SSVEP amplitudes averaged over all subjects and attentional conditions for each of the four frequencies. Electrodes used for the analysis are indicated by larger dots. $\boldsymbol{C}$, Grand-average amplitude spectrum for attend-different conditions in which either orange and magenta or violet and cyan were attended. D, Same as $\boldsymbol{C}$, but for conditions in which orange and cyan or violet and magenta were attended. Amplitudes at all four frequencies show clear enhancement by attention $(\boldsymbol{C}, \boldsymbol{D})$. Note that in half of all trials, the assignment of colors to sides and frequencies was swapped (cyan left, $7.5 \mathrm{~Hz}$; magenta left, $10 \mathrm{~Hz}$; violet right, $12 \mathrm{~Hz}$; orange right, $8.6 \mathrm{~Hz}$ ). These conditions showed a corresponding pattern of results and are included in the statistical analyses.

control condition to ensure that this test had sufficient power to detect a correlation of SSVEP amplitudes if indeed participants had switched attention between stimuli. For each pair of cued stimuli, the corresponding control condition was generated by combining all trials from the two conditions in which only one of the cued stimuli of the original condition was attended. For example, to generate a control condition for the condition "attend orange $(10 \mathrm{~Hz}$, left $)$ and cyan $(12 \mathrm{~Hz}$, right)," all trials of the conditions "attend orange and magenta" and "attend violet and cyan" were combined (for further explanation, see Results).

Statistical testing. Statistical tests (except for P3 latencies; see above) were performed by means of two-tailed $t$ tests. For direct comparisons between Experiments 1 and 2, two-sample $t$ tests assuming unequal variance between samples were used.

\section{Results}

Experiment 1: behavioral data

Observer sensitivity $\left(d^{\prime}\right)$ for discriminating target from distractor stimuli was higher when the same colors were attended in both visual fields compared to when opposite colors were attended $\left(t_{(12)}=8.2, p<10^{-5}\right.$; Table 1$)$. In both cases, performance was well above the chance level of $d^{\prime}=0$ (both $t_{(12)} \geq 10.3$, $\left.p<10^{-6}\right)$. Response bias $(C)$, a measure of participants tendency to respond to targets and distractors, did not differ from zero in either the attend-same or attendopposite condition (both $\left|t_{(12)}\right| \leq 1.46$, $p>0.1)$ and did not differ between these conditions $(|t| \leq 1.5, p>0.1)$. Reaction times to luminance decrement targets were faster in the attend-same than in the attend-opposite condition $\left(t_{(12)}=-10.4\right.$, $\left.p<10^{-6}\right)$.

Experiment 1: electrophysiological data A cluster of 11 occipital and parietal electrodes that exhibited the highest SSVEP amplitudes (Fig. 2B) was chosen for analysis. In conditions where the same colors were attended on both sides, there was clear enhancement of SSVEP amplitudes for each of the four frequencies when the driving stimulus was attended versus unattended (all $t_{(12)} \geq 3.23$, all $p<0.01$; Fig. $2 C)$. However, when opposite colors were attended there was no attentional modulation of SSVEP amplitudes (all $\left|t_{(12)}\right|<$ 0.91 , all $p>0.1$; Fig. $2 D$ ).

\section{Experiment 2}

To test whether the observed lack of attentional modulation of the SSVEP in attendopposite conditions in Experiment 1 might simply be explained by an inability to focus attention on two different colors at the same time, Experiment 2 was conducted with four different colors as described above (Fig. 3A).

Compared with Experiment 1, the attend-different conditions in Experiment 2 yielded intermediate values for $d^{\prime}$ (attend same vs attend different, $t_{(18.8)}=$ 2.33, $p<0.05$; attend different vs attend opposite, $\left.t_{(17.2)}=2.18 ; p<0.05\right)$. Response bias $(C)$ in the attend-different conditions did not differ from zero or from the conditions of Experiment 1 (all $|t| \leq 0.69, p>0.1$ ). Reaction times were faster than in the attend-opposite condition of Experiment $1\left(t_{(21.9)}=\right.$ $-2.69, p<0.05)$ but did not differ from the attend-same condition $\left(t_{(22)}=-0.50, p>0.1\right.$; Table 1$)$.

SSVEP amplitudes in Experiment 2 (Fig. 3B) showed a highly similar topographical distribution to those of Experiment 1 (Fig. $2 B)$. Accordingly, the same 11 electrodes were chosen for analysis. To assess attentional enhancement of SSVEP amplitudes when different colors were attended on the two sides in Experiment 2, amplitudes elicited at each frequency separately were averaged over the four conditions in which that frequency was attended and the four in which it was unattended. There was clear enhancement of SSVEP amplitudes elicited by the attended-color array for all four frequencies (all $t_{(10)} \geq 2.55$, all $p<0.05$; Fig. $3 C, D$ ).

To test whether this attentional modulation in Experiment 2 was due to concurrent selection of the two cued colors, or 
Table 1. Mean values and SEMs for behavioral measures of target detection performance under different attentional conditions

\begin{tabular}{|c|c|c|c|}
\hline & \multicolumn{2}{|l|}{ Experiment 1} & \multirow{2}{*}{$\begin{array}{l}\text { Experiment } 2 \\
\text { Different }\end{array}$} \\
\hline & Same & Opposite & \\
\hline Hit rate & $91.5(1.6)$ & $80.0(3.0)$ & $87.6(3.2)$ \\
\hline False alarms & $7.9(1.7)$ & $15.0(3.1)$ & $10.3(1.3)$ \\
\hline Sensitivity $d^{\prime}$ & $3.111(0.176)$ & $2.133(0.208)$ & $2.637(0.101)$ \\
\hline response bias C & $0.021(0.043)$ & $0.101(0.069)$ & $0.027(0.082)$ \\
\hline reaction time (ms) & 534.5 (12.7) & $586.8(11.6)$ & $543.0(11.4)$ \\
\hline
\end{tabular}

Data for attend-same and attend-opposite conditions of Experiment 1 and for attend-different conditions of Experiment 2 are shown.

whether it could be explained by averaging over trials in which participants alternated between attending to the cued colors on the left and on the right, SSVEP amplitudes from single trials were examined. For example, if only the cued color on the left was attended in some trials, then the SSVEP amplitude at the frequency of the cued stimulus on the left should show enhancement, while the frequency of the cued stimulus on the right should not. Conversely, trials where only the cued stimulus on the right was attended would show the opposite pattern. Hence, if participants switched between attending to the cued color on the left and the cued color on the right on different trials, the correlation of the SSVEP amplitudes of those two stimuli over all trials of that cue condition should be negative (for a similar analysis, see Andersen et al., 2008). However, the correlation between the single-trial SSVEPs to the two cued dot arrays in Experiment 2 did not differ from zero $\left(r=0.0035, t_{(87)}=0.161, p>0.1\right)$. This result was not simply due to insufficient power to detect such a negative correlation: for each cued pair of colors, a control analysis was performed on the combined set of single trials from the two different conditions in which only one of those colors was cued. In this case, the resulting overall correlation between the single SSVEP amplitudes of the two colors in the cued pair was indeed significantly negative $\left(r=-0.0438, t_{(87)}=-2.694, p<\right.$ $0.01)$. It is still conceivable, however, that participants shifted attention between the two cued colors in Experiment 2 within trials. If this were the case, reaction times for correct responses should show a bimodal distribution, with fast responses to targets of the currently attended color and slow responses to targets of the currently unattended color. Such a bimodal distribution was not apparent from visual inspection of all individual reaction time distributions. Additionally, average reaction times should be longer if participants shifted attention between the two cued stimuli compared to when they did not. However, reaction times in Experiment 2 were not slower than in the attend-same condition of Experiment 1 (Table 1). Accordingly, we conclude that participants were in fact able to attend concurrently to two different colors in the left and right visual fields.

To compare SSVEP amplitude modulations under the three attentional conditions (attend same and attend opposite from Experiment 1; attend-different from Experiment 2), SSVEP amplitudes were normalized by dividing by the mean amplitude over all attentional conditions and then collapsing across frequencies, as in previous studies (Andersen et al., 2008, 2011a). As in the analysis of the separate frequencies, attend-same and attend-different conditions exhibited clear SSVEP enhancement for the attended versus unattended dot arrays, while the attendopposite condition did not (attend same, $t_{(12)}=7.68, p<10^{-5}$; attend different, $t_{(10)}=6.96, p<10^{-4}$; attend opposite, $t_{(12)}=$ $0.14, p>0.1$; Figure 4 ). Direct comparison of the magnitude of these SSVEP attention effects revealed that attentional modulations (attended minus unattended amplitudes) were larger in

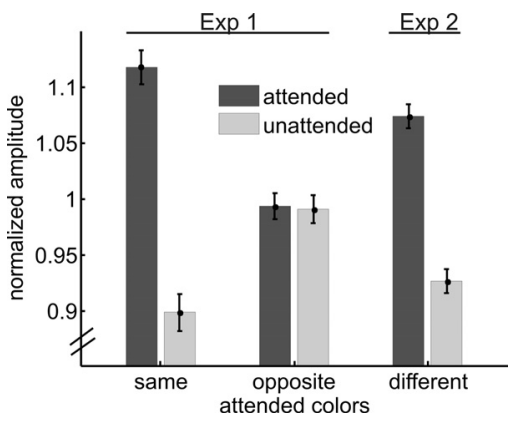

Figure 4. Normalized SSVEP amplitudes. Amplitudes were collapsed across frequencies after normalization for both experiments. Attend-same and attend-different conditions show clear attentional enhancement of SSVEP amplitudes, whereas attend-opposite conditions do not. Error bars represent the SEM.

attend-same and attend-different conditions than in the attendopposite condition (same vs opposite, $t_{(12)}=7.99, p<10^{-5}$; different vs opposite, $\left.t_{(21.4)}=5.00, p<10^{-4}\right)$. The magnitude of the attention effect on the SSVEP in the attend-same condition was marginally larger than in the attend-different condition $\left(t_{(21.1)}=2.03, p=0.055\right)$. In a previous study, SSVEPs revealed qualitatively different attention effects at early and mid levels of visual processing (Andersen et al., 2012). However, no such pattern was apparent in the present paradigm. When the above analysis was repeated for the six occipital and inferior $\left(\mathrm{O} 1, \mathrm{Oz}, \mathrm{O}_{2}, \mathrm{I} 1\right.$, $\mathrm{Iz}, \mathrm{I} 2)$ electrodes and the five parietal (PO7, PO3, POz, PO4, PO8) electrodes separately, both clusters showed an equivalent pattern of results as the cluster of all 11 electrodes used in the main analysis.

\section{ERPs to targets and distractors}

The SSVEP was used to quantify processing in early visual areas. To gain information on later processing stages, we analyzed ERPs elicited by target and distractor events in both experiments. The main focus was on the late positive (P3 or P300) component, which varies in amplitude and latency according to the accuracy and confidence with which task-relevant stimuli are detected (Squires et al., 1973; Hillyard and Picton, 1987; Polich, 2007). To determine the time of onset of attentional modulation of the P3, we subjected the difference between target (attended) and distractor (unattended) waveforms averaged over the different stimuli to a running $t$ test for each of the three attention conditions. Significantly larger P3 amplitudes for targets as opposed to distractors were observed beginning from $367 \mathrm{~ms}$ onward for attend-same, from $449 \mathrm{~ms}$ for attend-opposite, and from $416 \mathrm{~ms}$ for attend-different conditions (Fig. 5). Corresponding to these differences in $\mathrm{P} 3$ onset latency, the latency of $\mathrm{P} 3$ peak modulation (i.e., the $50 \mathrm{~ms}$ window with the largest difference between attended and unattended amplitudes) was earlier for attend-same than for attend-opposite conditions (465 vs $523 \mathrm{~ms}, p<0.05$ ) and intermediate for attend-different conditions (508 ms, same vs different, $p=0.057$; opposite vs different, $p>0.1)$. Attentional modulation of P3 peak amplitude was highly significant for all three attention conditions (all $t>3.96$, all $p<0.005$ ) with attend-same conditions showing larger modulation than attendopposite conditions $\left(t_{(12)}=3.07, p<0.01\right)$. Attend-different conditions showed intermediate modulation, which did not differ significantly from either of the two other conditions (both $|t| \leq 0.949, p>0.1)$. No other ERP components to target and distractor events were evident, as these were masked by the ongoing SSVEP. 


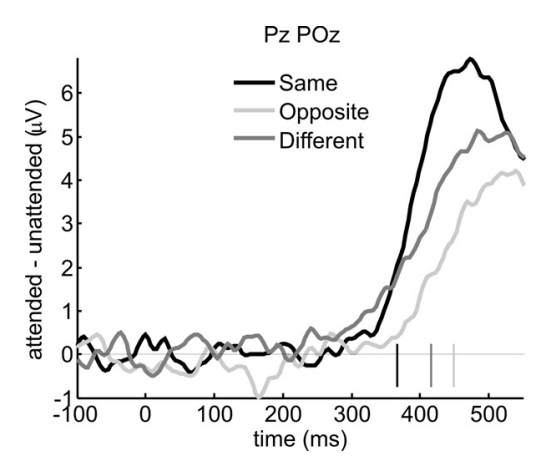

Figure 5. ERPs to targets and distractors. Difference waveforms formed by subtracting ERPs elicited by luminance decrement distractors (unattended) from those elicited by physically identical targets (attended), averaged over electrodes $\mathrm{Pz}$ and $\mathrm{POz}$. Onsets of significant targetdistractor differences are indicated by short vertical lines. Attentional modulation of the P3 component was the strongest and occurred earliest for attend-same conditions, and was latest and smallest for attend-opposite conditions. Attend-different conditions (Experiment 2) showed intermediate values. This pattern is consistent with behavioral performance as measured by sensitivity $d^{\prime}$ and reaction time (Table 1).

\section{Discussion}

Our results support the hypothesis that different colors can be concurrently attended, but that this ability to divide attention is constrained by obligatory global enhancement of feature selective processing. When dot arrays of the same color were attended in the left and right visual fields (Experiment 1), there was a clear enhancement of SSVEP amplitudes to the attended arrays together with fast and accurate detections of the luminancedecrement targets. In contrast, when opposite colors were attended in the left and right fields, SSVEP amplitudes did not differ between the attended and unattended dot arrays, and target detections were slower and less accurate. The SSVEP amplitudes in these attend-opposite conditions were intermediate between the amplitudes elicited by attended and unattended stimuli in the attend-same conditions. These results follow directly from the hypothesis that attending to opposing colors in the left and right visual fields led to cancellation of selectivity of attended and unattended colors due to the spatially global spread of attention. Moreover, SSVEP recordings showed that selectivity was completely cancelled in the attend-opposite condition, which implies that the global feature enhancement was of equal magnitude across the visual field (i.e., uniform) rather than a decrementing spread from the attended location(s). The results of Experiment 2 further showed that SSVEPs were selectively enhanced and behavioral performance was improved for attended pairs of different colors when the overlapping unattended colors were not being attended in the opposite field. Together, our results reveal a fundamental property of feature-selective attention, namely, that attended features are globally facilitated throughout the visual field, even when such facilitation conflicts with the task demands and is actually detrimental to performance.

Previous SSVEP studies have provided evidence for significant independence of attentional selection on the basis of location and color. In particular, attentional selection of color was found to occur under circumstances that strictly rule out a mediation by spatial attention (Andersen et al., 2009). Moreover, when selection was based on both spatial position and color, each attribute contributed independently to the modulation of stimulus processing (Andersen et al., 2011a). The present study adds to this evidence by showing that attentional selection of color cannot be restricted to specific spatial locations, even when the task at hand explicitly demands it. These observations extend the scope of the feature-similarity gain model (Treue and Martínez Trujillo, 1999; Maunsell and Treue, 2006), which proposes that attentional selections of different features are combined additively and that feature-based attention globally affects the sensory gain of neurons with receptive fields throughout the visual field, regardless of the location of the relevant, attended stimuli. Based on the present results, we conclude that this global feature enhancement is an invariant property of human vision that cannot be overridden by task demands. The present SSVEP recordings further demonstrate that this obligatory global feature selection and facilitation occurs at an early level of visual cortical processing. The attend-opposite conditions of Experiment 1 also critically test the (neural) theory of visual attention (TVA; Bundesen, 1990; Bundesen et al., 2005), which, like the feature-similarity gain model, assumes that the attentional valence of an object is the weighted sum over different categories (i.e., feature dimensions). Due to the global effect of attentional weights in this model, any feature-selective enhancement in the attend-opposite conditions would have violated the weight equation of TVA. Hence, the observed lack of selectivity in the attend-opposite conditions is fully consistent with the TVA.

Target detection performance remained well above chance levels in all conditions, which rules out the possibility that the absence of early attentional modulation found in the attendopposite condition was due to failure of effort by the participants. Evidently, selective target discrimination processes took place at a later decision stage reflected in the P3 component of the ERPs to targets and distracters. This target discrimination may have benefited from the enhanced early sensory gain in the attend-same and attend-different conditions (reflected in the SSVEP), resulting in larger P3 amplitudes and shorter P3 latencies in association with better target detection performance. The lack of any change in participants' response bias $(C)$ gives a further indication that their decision strategy did not change across conditions. The present behavioral results are in agreement with the previous study of Saenz et al. (2003), who also found that attending to the same feature at two different locations results in better performance than attending to opposing features (Lo et al., 2012). The present SSVEP and ERP recordings provide critical new information about the mechanism of this deficient target detection under attend-opposite conditions, namely, the complete cancellation of an early sensory gain benefit can be ascribed to the global and uniform enhancement of attended feature processing across the entire visual field.

Our conclusion that feature selection is spatially uniform is based on the full cancellation of selectivity at a symmetrical position in the opposite visual field in the attend-opposite conditions of Experiment 1. However, the spread of feature-based attention is not restricted to such symmetrical left/right locations (Serences and Boynton, 2007). Accordingly, our conclusion must be qualified in that we did not systematically vary the spatial position of stimuli to test whether it generalizes to all locations.

Alternative explanations of the present SSVEP results that assume attentional selections might have been based on other factors such as depth or luminance cues, flicker frequencies, or interference from targets and distractors can be ruled out. All dots were drawn in random order, thereby precluding any systematic occlusion of dots of one color by dots of a different color, which might otherwise have induced a depth cue. The idea that selection was based on flicker frequencies rather than color is inconsistent with the present results and those of previous studies (Andersen et al., 2008, 2011a). If this had been the case, attentional modulation should also have been observed in attend- 
opposite trials, since flicker frequencies always differed between the four stimuli. Isoluminance of dots of all colors to the gray background was adjusted individually, and only trials without luminance decrement targets and distractors were entered into the SSVEP analysis, thereby ruling out any influence of luminance differences or processes related to target and distractor processing on our measures of attentional selection. Finally, the analysis of single-trial amplitude modulations of the SSVEP in Experiment 2 showed that subjects were capable of attending concurrently to different noncompeting colors in the left and right fields and did not alternate attention from one to the other.

Studies in which stimuli were presented transiently have generally not found evidence for global feature facilitation (Hillyard and Münte, 1984; Anllo-Vento and Hillyard, 1996; Festman and Braun, 2010). Under such conditions, the ERP signature of feature selection was found to be a selection negativity component beginning around $150 \mathrm{~ms}$ after stimulus onset, which was evident to a much greater extent for stimuli at attended locations than at unattended locations (Anllo-Vento and Hillyard, 1996). By comparison, in studies where stimuli were presented continuously, ERPs elicited by irrelevant probe flashes at an unattended location showed feature-selective enhancement of the P1 or N1 components, which peak at around 100 or $170 \mathrm{~ms}$ after stimulus onset, respectively (Zhang and Luck, 2009; Bondarenko et al., 2012). Like the SSVEP modulations recorded in the present study, the P1 and N1 have been proposed to reflect a gain enhancement of visual processing of attended stimuli (Hillyard and Anllo-Vento, 1998). Further evidence comes from neurophysiological studies in monkeys (Treue and Martínez Trujillo, 1999) and fMRI studies in humans (Saenz et al., 2002), in which global feature gain effects were evident under conditions of continuous stimulus presentation. Together, these observations suggest that the attentional mechanism leading to global feature gain enhancement in visual cortex can only be engaged when stimuli are continuously visible for sufficiently long periods, perhaps of the order of seconds. With shorter stimulus presentation times, this global enhancement mechanism may not have sufficient time to "tune in" to the relevant feature, and feature selection then only occurs at later stages, at which it is not spatially global. The operation of these later, spatially localized stages of feature selection were evident in the present experiment in the behavioral responses and P3 amplitudes elicited by target and distractor events.

In summary, the present study shows that attention can be deployed to two different colors at the same time and confirms previous findings of a global spread of feature-selective attention throughout the visual field, which enhances attended-feature inputs in visual cortex. The present findings extend previous formulations by showing that the spatially global spread of feature selection at early levels of visual processing is a fundamental and obligatory mechanism of attention that is engaged even when it explicitly conflicts with task demands. Moreover, the gain facilitation of an attended feature appears to be equal in magnitude at both attended and unattended locations (i.e., uniform), at least for mirror-symmetric left/right locations. These properties of visual attention impose severe constraints on the ability to attend to a particular feature at only a single location.

\section{References}

Andersen SK, Hillyard SA, Müller MM (2008) Attention facilitates multiple stimulus features in parallel in human visual cortex. Curr Biol 18:10061009. CrossRef Medline

Andersen SK, Müller MM, Hillyard SA (2009) Color-selective attention need not be mediated by spatial attention. J Vis 9(6):2 1-7. CrossRef
Andersen SK, Fuchs S, Müller MM (2011a) Effects of feature-selective and spatial attention at different stages of visual processing. J Cogn Neurosci 23:238-246. CrossRef Medline

Andersen SK, Müller MM, Hillyard SA (2011b) Tracking the allocation of attention in visual scenes with steady-state evoked potentials. In: Cognitive neuroscience of attention, Ed 2 (Posner MI, ed), pp 197-216. New York: Guilford.

Andersen SK, Müller MM, Martinovic J (2012) Bottom-up biases in feature-selective attention. J Neurosci 32:16953-16958. CrossRef Medline

Anllo-Vento L, Hillyard SA (1996) Selective attention to the color and direction of moving stimuli: electrophysiological correlates of hierarchical feature selection. Percept Psychophys 58:191-206. CrossRef Medline

Awh E, Pashler H (2000) Evidence for split attentional foci. J Exp Psychol Hum Percept Perform 26:834-846. CrossRef Medline

Bondarenko R, Boehler CN, Stoppel CM, Heinze HJ, Schoenfeld MA, Hopf JM (2012) Separable mechanisms underlying global feature-based attention. J Neurosci 32:15284-15295. CrossRef Medline

Bundesen C (1990) A theory of visual attention. Psychol Rev 97:523-547. CrossRef Medline

Bundesen C, Habekost T, Kyllingsbaek S (2005) A neural theory of visual attention: bridging cognition and neurophysiology. Psychol Rev 112:291328. CrossRef Medline

Delorme A, Makeig S (2004) EEGLAB: an open source toolbox for analysis of single-trial EEG dynamics including independent component analysis. J Neurosci Methods 134:9-21. CrossRef Medline

Di Russo F, Teder-Sälejärvi W, Hillyard SA (2002) Steady-state VEP and attentional visual processing. In: The cognitive electrophysiology of mind and brain (Zani A, Proverbio AM, eds), pp 259-273. New York: Academic.

Festman Y, Braun J (2010) Does feature similarity facilitate attentional selection? Atten Percept Psycho 72:2128-2143. CrossRef

Hayden BY, Gallant JL (2009) Combined effects of spatial and feature-based attention on responses of V4 neurons. Vision Res 49:1182-1187. CrossRef Medline

Hillyard SA, Anllo-Vento L (1998) Event-related brain potentials in the study of visual selective attention. Proc Natl Acad Sci U S A 95:781-787. CrossRef Medline

Hillyard SA, Münte TF (1984) Selective attention to color and location: an analysis with event-related brain potentials. Percept Psychophys 36:185198. CrossRef Medline

Hillyard SA, Picton TW (1987) Electrophysiology of cognition. In: Handbook of physiology (Plum F, ed), pp 519-584. Bethesda, MD: American Physiological Society.

Junghöfer M, Elbert T, Tucker DM, Rockstroh B (2000) Statistical control of artifacts in dense array EEG/MEG studies. Psychophysiology 37:523532. CrossRef Medline

Kristjánsson A (2006) Simultaneous priming along multiple feature dimensions in a visual search task. Vision Res 46:2554-2570. CrossRef Medline

Lo SY, Howard CJ, Holcombe AO (2012) Feature-based attentional interference revealed in perceptual errors and lags. Vision Res 63:20-33. CrossRef Medline

Lu J, Itti L (2005) Perceptual consequences of feature-based attention. J Vis 5(7):622-631.

Maljkovic V, Nakayama K (1994) Priming of pop-out: I. Role of features. Mem Cognit 22:657-672. CrossRef Medline

Martinez-Trujillo JC, Treue S (2004) Feature-based attention increases the selectivity of population responses in primate visual cortex. Curr Biol 14:744-751. CrossRef Medline

Maunsell JH, Treue S (2006) Feature-based attention in visual cortex. Trends Neurosci 29:317-322. CrossRef Medline

Melcher D, Papathomas TV, Vidnyánszky Z (2005) Implicit attentional selection of bound visual features. Neuron 46:723-729. CrossRef Medline

Morgan ST, Hansen JC, Hillyard SA (1996) Selective attention to stimulus location modulates the steady-state visual evoked potential. Proc Natl Acad Sci U S A 93:4770-4774. CrossRef Medline

Müller HJ, Heller D, Ziegler J (1995) Visual search for singleton feature targets within and across feature dimensions. Percept Psychophys 57:117. CrossRef Medline

Müller MM, Malinowski P, Gruber T, Hillyard SA (2003) Sustained division of the attentional spotlight. Nature 424:309-312. CrossRef Medline

Polich J (2007) Updating P300: an integrative theory of P3a and P3b. Clin Neurophysiol 118:2128-2148. CrossRef Medline 
Pylyshyn ZW, Storm RW (1988) Tracking multiple independent targets: evidence for a parallel tracking mechanism. Spat Vis 3:179-197. CrossRef Medline

Saenz M, Buracas GT, Boynton GM (2002) Global effects of feature-based attention in human visual cortex. Nat Neurosci 5:631-632. CrossRef Medline

Sàenz M, BuraĉasGT, Boynton GM (2003) Global feature-based attention for motion and color. Vision Res 43:629-637. CrossRef Medline

Serences JT, Boynton GM (2007) Feature-based attentional modulations in the absence of direct visual stimulation. Neuron 55:301-312. CrossRef Medline

Squires KC, Hillyard SA, Lindsay PH (1973) Vertex potentials evoked during auditory signal detection: Relation to decision criteria. Percept Psychophys 14:265-272. CrossRef

Stoppel CM, Boehler CN, Sabelhaus C, Heinze HJ, Hopf JM, Schoenfeld MA (2007) Neural mechanisms of spatial- and feature-based attention: a quantitative analysis. Brain Res 1181:51-60. CrossRef Medline

Stoppel CM, Boehler CN, Strumpf H, Krebs RM, Heinze HJ, Hopf JM, Schoe- nfeld MA (2012) Spatiotemporal dynamics of feature-based attention spread: evidence from combined electroencephalographic and magnetoencephalographic recordings. J Neurosci 32:9671-9676. CrossRef Medline

Treue S, Martínez Trujillo JC (1999) Feature-based attention influences motion processing gain in macaque visual cortex. Nature 399:575-579. CrossRef Medline

Wagner G, Boynton RM (1972) Comparison of four methods of heterochromatic photometry. J Opt Soc Am 62:1508-1515. CrossRef Medline

Wickens TD (2002) Elementary signal detection theory. New York: Oxford UP.

Wolfe JM (1994) Guided Search 2.0: A revised model of visual search. Psychon Bull and Rev 1:202-238. CrossRef

Zhang W, Luck SJ (2009) Feature-based attention modulates feedforward visual processing. Nat Neurosci 12:24-25. CrossRef Medline

Zhou H, Desimone R (2011) Feature-based attention in the frontal eye field and area V4 during visual search. Neuron 70:1205-1217. CrossRef Medline 